FORMATION Formation emploi

Revue française de sciences sociales

120 | Octobre-Décembre 2012

Parcours de formation : la recomposition des

ségrégations

\title{
Parcours scolaires en France et espace d'opportunités : une analyse à l'aune de la théorie des capabilités de Sen
}

Education trajectories and 'opportunity sets': a study in the light of Sen's theory

of capabilities

Schulische Laufbahnen in Frankreich und Raum für Opportunitäten: eine

Analyse nach dem Capacity Approach von Amartya Sen

Itinerarios escolares en Francia y espacio de oportunidades : un análisis según la teoría de las capacidades de Sen

Valérie Germain et Noémie Olympio

\section{OpenEdition \\ Journals}

Édition électronique

URL : http://journals.openedition.org/formationemploi/3754

DOI : 10.4000/formationemploi.3754

ISSN : 2107-0946

Éditeur

La Documentation française

Édition imprimée

Date de publication : 31 octobre 2012

Pagination : 13-33

ISSN : 0759-6340

Référence électronique

Valérie Germain et Noémie Olympio, «Parcours scolaires en France et espace d'opportunités : une analyse à l'aune de la théorie des capabilités de Sen », Formation emploi [En ligne], 120 | OctobreDécembre 2012, mis en ligne le 26 février 2013, consulté le 30 octobre 2020. URL : http://

journals.openedition.org/formationemploi/3754; DOI : https://doi.org/10.4000/formationemploi.3754 


\title{
Parcours scolaires en France et espace d'opportunités : une analyse à l'aune de la théorie des capabilités de Sen
}

\begin{abstract}
NoÉMIE OlYMPIO
Doctorante en économie, sous la direction d'Eric Verdier et Vanessa Di Paola, au Laboratoire d'économie et de sociologie du travail (LEST-

CNRS, Aix-en-Provence)

VALÉRIE GERMAIN Doctorante en sociologie, sous la direction de Thierry Blöss, au Laboratoire d'économie et de sociologie du travail (LEST-CNRS, Aix-en-Provence)
\end{abstract}

Résumé

Parcours scolaires en France et espace d'opportunités : une analyse à l'aune de la théorie des capabilités de Sen

De nombreuses études ont mis en exergue le poids de l'environnement socio-culturel sur les trajectoires scolaires. Il s'agit ici d'analyser comment les différents facteurs sociaux, qui peuvent se cumuler, conduisent à élargir, pour certains, le champ des possibles et à le restreindre, pour d'autres. Nous proposons d'analyser l'espace des possibles au cours des trajectoires de formation à l'aune de la théorie des " capabilités » de l'économiste Amartya Sen. Appliquée à l'éducation, elle contribue à renouveler l'analyse de l'égalité des chances à l'école en se focalisant sur les opportunités réelles des élèves au cours de leur trajectoire de formation et sur les contraintes qu'ils peuvent rencontrer.

Mots clés : Origine sociale, cheminement scolaire, approche par les capabilités, inégalités

Abstract

Education trajectories and 'opportunity sets' : a study in the light of Sen's theory of capabilities

Many studies highlighted the impact of socio-cultural background on educational trajectories. The purpose of this article is to analyze how the different social factors lead to the multiplication of opportunities for some individuals while restraining the opportunities for others. We analyze opportunities in educational trajectories in the light of Sen's theory of capabilities. When applied to education, this theory renews the 
debate on equality of opportunity by focusing on students' real opportunities and on the constraints they might face.

Key words : Social origin, school paths, capabilities approach, inequalities

Journal of Economic Literature: I 29, J 24

Traduction : Auteurs

Tout système éducatif s'inscrit dans un contexte sociétal (Maurice et al., 1982) spécifique, et celui de la France n'échappe pas à la règle. Selon la typologie élaborée par Mons (2004), le système éducatif français se caractérise par un modèle d'intégration uniforme. Une des caractéristiques de ce modèle est un tronc commun long. Pour autant, des classes de niveau sont organisées de manière informelle ; de même, tous les établissements d'enseignement ne jouissent pas de la même réputation. Dans le prolongement des travaux de Mons, Verdier (2008), en s'intéressant aux régimes d'action en matière de formation des pays européens, souligne que la France se caractérise par une prédominance du régime académique (donc par une certaine sur-valorisation des savoirs académiques). Ainsi la formation professionnelle en France est souvent perçue comme une formation de "seconde zone ", destinée à accueillir les élèves aux plus faibles résultats scolaires. De nombreuses études, celles de Bourdieu (1970) ou de Duru-Bellat (2002) pour n'en citer que deux, ont mis en exergue le poids de l'environnement socio-culturel sur les trajectoires scolaires au cours de l'enseignement secondaire. Ce poids de l'environnement socio-culturel reste prégnant dans l'enseignement supérieur.

Les différents facteurs sociaux peuvent se cumuler, élargissant pour certains le champ des possibles et le restreignant pour d'autres. Cet espace des possibles au cours des trajectoires de formation sera analysé à l'aune de la théorie des "capabilités » de l'économiste Amartya Sen. Cette théorie a pour particularité de s'intéresser aux marges de manœuvre que peuvent avoir les personnes. D’une manière générale, la théorie de Sen met l'accent sur les opportunités réelles dont peuvent disposer les individus de choisir des parcours de vie auxquels ils attribuent de la valeur. Appliquée à l'éducation, elle contribue à renouveler l'analyse de l'égalité des chances à l'école. Si Boudon se focalise sur la rationalité des acteurs en arguant que les choix scolaires résultent de stratégies individuelles, la théorie de Bourdieu souligne le poids de l'origine sociale dans les inégalités scolaires en raison de la proximité culturelle qui existe entre la classe dominante et l'école. La théorie de Sen permet de s'intéresser aux opportunités réelles des élèves au cours de leurs trajectoires de formation et sur les contraintes qu'ils peuvent rencontrer. Elle permet notamment d'analyser l'espace de choix de l'individu et de différencier les choix réels des choix contraints.

L'analyse de la liberté de choix des personnes et de leur espace des possibles nécessitant une approche dynamique, la partie empirique de ce travail est réalisée grâce à l'enquête longitudinale de la Direction de la Prospective et de la Performance (DEPP) du ministère 
de l'Éducation nationale. Ce panel a suivi les trajectoires de formation des jeunes, de leur entrée en sixième jusqu’à leur entrée dans la vie active. En s'appuyant sur la théorie de Sen, nous proposons une typologie des trajectoires de formation et d'insertion. Pour chacune d'entre elles, nous tentons d'apprécier d'une part, les déterminants de l'appartenance aux différentes trajectoires-types et d'autre part, l'espace des possibles associé à chaque trajectoire.

Dans une première partie, nous présentons le cadre théorique dans lequel s'inscrit cet article. Dans une seconde partie, notre objectif est de mettre à jour la typologie des trajectoires de formation. Enfin, la dernière partie met à l'épreuve notre modèle multinomial.

\section{La théorie des « capabilités » pour analyser des situations d'inégalités dans l'éducation}

Le lien entre éducation et "capabilités " paraît en fait assez naturel, l'éducation apparaissant comme un vecteur incontournable de l'augmentation des possibilités réelles des personnes.

Les « capabilités » d'une personne représentent l'ensemble des libertés d'accomplir permettant d'augmenter la qualité de vie. Une distinction fondamentale doit être opérée entre accomplissement et liberté d'accomplir. Les accomplissements représentent ce qui a été réalisé par l'individu, comme un niveau d'éducation atteint. Sen $(1985,1992,1993)$ les nomme plus communément les "fonctionnements ". Les libertés d'accomplir sont les possibilités de choisir entre différentes options ; elles représentent les «capabilités » des individus. Ainsi, évaluer uniquement la situation des personnes (ou leur « fonctionnement ") ne nous indique pas si elles avaient au départ les mêmes opportunités, le même espace de choix.

Les données disponibles n'offrent généralement pas d'indicateurs de liberté de choix permettant de renseigner sur l'ensemble des alternatives possibles, des possibilités formelles et des possibilités réellement atteignables. Face à cette difficulté, Sen propose un « compromis pratique " : partir des données existantes, cerner les informations renseignant sur les opportunités de choix et les contraintes des personnes et analyser ces situations selon le cadre analytique des « capabilités ».

La théorie de Sen constitue une grille d'analyse stimulante et novatrice du point de vue de la justice sociale ; elle s'intéresse en effet aux opportunités réelles dont disposent les individus au cours de leur vie. Selon Sen, les inégalités à prendre en compte ne sont pas seulement les inégalités en termes de manque de ressources ou de moyens mais également les inégalités en termes de manque d'options, de possibilités ou d'opportunités réelles. 
Cette théorie nous permet de repenser l'inégalité face à l'éducation. L’ensemble " capabilités » reflète, dans l'espace des fonctionnements, la liberté qu'une personne a de choisir entre différentes options. En effet, il existe une grande différence entre le fait de suivre une certaine formation par choix véritable ou par contrainte. Par exemple, ce n’est pas la même chose du point de vue de la liberté de choix d'opter pour une formation professionnelle courte par contrainte (après une décision de conseil de classe) ou par vocation. Pour autant, dans les deux cas, le résultat est le même. Ce n’est également pas la même chose de choisir un parcours universitaire court en raison d'une aversion pour les études longues que de choisir un tel parcours par manque de ressources. Ainsi ce qui paraît injuste, du point de vue de la théorie de Sen, ce ne sont pas tant des inégalités de résultats que des inégalités à la base, dans l'espace des possibles des personnes.

Cette théorie nous autorise alors à considérer les caractéristiques personnelles et sociétales influençant les choix. Ce dernier aspect est important car les préférences sont souvent prédéterminées par la position sociale et culturelle de l'individu (Otto et Ziegler, 2006 ; Bourdieu et Passeron, 1970). La théorie de Sen permet ainsi la prise en compte des relations sociales, des contraintes et opportunités survenant dans les trajectoires en fonction des structures sociétales et institutionnelles (Robeyns, 2000).

Nous nous focalisons sur les trajectoires des individus car une analyse en termes de " capabilités » nécessite une approche dynamique. Dans la partie empirique, nous optons alors pour une analyse par trajectoire-type. En effet, l'évaluation des " capabilités " des personnes requiert d'analyser les espaces d'opportunités et de contraintes qui s'ouvrent et se ferment dans le temps. Une situation contrainte au départ peut déboucher sur une situation ouvrant davantage de possibilités plus tard. Dans une perspective purement senienne, en suivant notamment Verhoeven et al. (2007), l'idéal serait de disposer de variable renseignant sur les alternatives possibles des différents choix éducatifs, ainsi que sur les possibilités formelles et les possibilités réellement atteignables pour les individus.

À défaut de disposer de telles données, nous nous concentrons ici sur la détermination des trajectoires et sur une approche en termes de réversibilité/irréversibilité de celles-ci. Cette dernière approche a notamment été évoquée par Verhoeven et $a l$. (2007) ; ils avaient alors démontré que les étudiants orientés vers les filières professionnelles revenaient très rarement vers une formation plus générale alors même que cette possibilité existe (du moins formellement). Dans notre étude, l'irréversibilité sera appréciée au regard des impacts des situations éducatives de départ sur la situation en 2007. Nous nous intéresserons non seulement à l'impact des choix éducatifs mais également à celui de la performance scolaire en début de parcours. Dès lors, si la performance scolaire à un instant $t$ et les choix scolaires en tout début de parcours sur-déterminent le parcours de formation et d'insertion, les parcours sont relativement irréversibles.

Les structures institutionnelles (rôle des notes, type de sélection) vont laisser plus ou moins de place au rôle de l'environnement familial sur le parcours de formation. Dans la lignée des travaux en économie de l'éducation comme ceux de Ammermüller (2005) et 
Woesmann (2004), nous examinons alors l'influence de l'environnement familial et plus globalement socio-économique et culturel de l'individu sur sa trajectoire de formation et d'insertion. Un modèle éducatif où le rôle de l'environnement familial de l'individu est déterminant sur la trajectoire de formation et d'insertion est un modèle où l'espace des possibles des individus est réellement limité. Un fort poids de l'environnement familial sur la trajectoire serait d'autant plus pénalisant qu'en France le type de formation détermine largement le type d'insertion mais également les possibilités de carrière et de formation continue. Cela signifierait que, pour certaines populations, l'espace des possibles est non seulement limité dans le temps mais surtout déterminé à la base.

Ainsi, à chaque trajectoire-type (présentées en détail dans la section suivante) sont associés un espace des possibles et des contraintes. Il s'agit plus précisément de nous intéresser, dans la partie qui suit, aux contraintes structurelles à l'intérieur des trajectoires de formation afin d'en déterminer leur caractère (subi ou choisi). L'entrée sur le marché du travail et, d'une manière plus générale, l'entrée dans la vie adulte des acteurs sociaux prolongent cette analyse.

\section{Trajectoires-types des parcours de formation et d'emploi des jeunes du panel}

Après avoir présenté les trajectoires-types des parcours de formation et d'emploi des jeunes du panel, nous démontrerons que tous les individus n'ont pas les mêmes chances d'appartenir à une trajectoire-type. Enfin, nous soulignerons que l'appartenance à une trajectoiretype n'ouvre pas les mêmes possibilités en termes de réalisations lors du passage à la vie adulte.

\subsection{Les parcours de formation : une analyse par trajectoire-type}

Une approche par trajectoires-types nous permet d'apprécier la dynamique individuelle de la trajectoire de formation et d'emploi. Ce dernier aspect est d'autant plus intéressant que l'analyse de l'espace des possibles des individus et de leur contrainte nécessite cette approche dynamique. 


\section{Encadré 1}

\section{Le panel DEPP-EVA (Direction de l'évaluation de la prospective et de la performance- Entrées dans la vie active)}

Ce travail s'appuie sur le panel de la Direction de l'évaluation de la prospective et de la performance (DEPP) du ministère de l'Éducation nationale de 1995. Ce panel a suivi un échantillon représentatif au 1/40 ème de jeunes entrés en sixième en 1995. II renseigne sur les parcours scolaires en observant les individus jusqu'à leur sortie du système éducatif. Les individus ont donc fait l'objet d'une interrogation annuelle où deux types d'informations sont recueillis : des informations sur la situation scolaire de l'élève et des informations sur les caractéristiques de l'établissement fréquenté. À côté de cette actualisation annuelle, le panel a été complété : - par une enquête auprès des familles en 1998, l'objectif étant d'approcher l'environnement familial de l'élève ;

- par une enquête sur la procédure d'orientation en fin de troisième ;

- par une enquête sur les projets des individus et l'estime de soi (l'enquête Jeunes 2002) ;

- par un suivi des jeunes dans l'enseignement supérieur (l'enquête SUP).

En outre, depuis 2004-2005, I'Insee, en collaboration avec la DEPP, a interrogé les jeunes du panel 95 qui n'étaient plus suivis par la DEPP, à travers un protocole d'enquête dénommé « Entrées dans la Vie Adulte EVA ». Nous disposons alors d'informations sur les parcours de formation et d'insertion jusqu'en 2007-2008. Lors de l'entrée en sixième, 17830 élèves ont été interrogés. 4274 individus ont été interrogés durant les deux années après le baccalauréat. Le panel EVA (à partir de la troisième année après le baccalauréat) a pu retrouver des personnes, notamment les individus ayant interrompu leurs études. En 2005, l'enquête EVA a porté sur 4219 personnes interrogées. Travaillant sur des trajectoires, nous avons choisi de prendre en considération les individus suivis par l'ensemble des trois panels. Au final, l'échantillon sur lequel nous travaillons se compose de 4218 individus.

En effet, afin d'étudier les trajectoires scolaires et professionnelles, nous avons sélectionné les individus ayant renseigné leur « situation » (études ou emploi) de la première année à la dernière année d'enquête, c'est-à-dire de 1995 à 2007.

Même si I'Insee est parvenu à récupérer une grande partie des jeunes qui n'étaient plus suivis par la DEPP (70\%), certaines années restent sans information (principalement entre 2003 et 2006) pour certains individus. C'est particulièrement le cas pour les individus sortis du système éducatif sans le bac et qui sont entrés directement sur le marché du travail avec un diplôme de niveau V (CAP, BEP : certificat d'aptitude professionnelle et brevet d'études professionnelles). Si certains de ces individus sont englobés dans les trajectoires professionnelles par le jeu de l'Optimal Matching (méthode d'appariement optimal, cf. encadré 2), une partie d'entre eux reste sous-représentée dans nos trajectoires.

Afin de caractériser l'environnement familial de l'individu, les variables socio-économiques et culturelles retenues sont le niveau d'éducation du père, sa nationalité, l'aide aux devoirs par la famille et l'adéquation entre les ressources de la famille et les études envisagées. Nous aurions voulu pouvoir davantage prendre en compte l'environnement culturel en utilisant des variables comme le nombre de livres dans le foyer ou les sorties culturelles de la famille ; à défaut, nous savons si l'individu est inscrit dans une bibliothèque ou non.

Pour les variables de choix, nous prenons en compte le premier vœu d'orientation formulé par la famille, le fait d'avoir été orienté ou non selon ses vœux dans son parcours et le motif d'arrêt des études (notamment pour appréhender si l'arrêt est subi ou choisi). En outre, il aurait été souhaitable de prendre en compte la satisfaction par rapport à la formation proposée mais nous disposons seulement ici de la perception qu'ont les individus de leur avenir professionnel.

Pour mesurer la qualité d'entrée dans la vie adulte, nous prenons en compte le type de contrat de travail, la situation face au chômage, la situation face au logement (le fait d'avoir un logement indépendant) et le fait d'avoir à faire face à des difficultés financières. Notons que d'autres dimensions importantes de la vie adulte (pour lesquelles nous ne disposons pas de variables) auraient été souhaitables dans une perspective « capabilités », comme le fait de posséder un véhicule motorisé ou la satisfaction par rapport à l'emploi ou au logement. 
Pour la construction de nos trajectoires, les individus peuvent se situer dans neuf situations différentes, qui sont les suivantes :

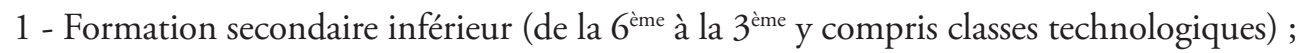
2 - Formation secondaire supérieur professionnel (court : $\mathrm{CAP}^{1}-\mathrm{BEP}^{2}$ et long : baccalauréat professionnel);

3 - Formation secondaire supérieur technologique ;

4 - Formation secondaire supérieur général ;

5 - Etudes supérieures ;

6 - Autres formations (formation continue diplômante post CAP ou post BEP) ;

7 - En emploi ;

8 - Sans emploi et à la recherche d'un emploi ;

9 - Autres situations professionnelles.

La procédure de classification retenue a permis de faire émerger cinq trajectoires-types :

1- Formation générale puis études supérieures longues ;

2- Formation technologique, études supérieures courtes puis entrée sur le marché du travail ;

3- Formation professionnelle, études supérieures courtes puis entrée sur le marché du travail ;

4- Formation générale, études supérieures courtes puis entrée sur le marché du travail ;

5- Formation professionnelle et technologique, études supérieures courtes puis entrée sur le marché du travail.

Ces cinq classes de trajectoires ont été construites en rapprochant les individus qui ont connu les mêmes situations aux mêmes moments. L'encadré 2 revient sur la méthode employée.

1. Certificat d'aptitude professionnelle.

2. Brevet d'études professionnelles. 


\section{Encadré 2}

\section{Méthode de classification employée}

Afin de créer les typologies, nous avons utilisé la Méthode d'Appariement Optimal (Optimal Matching) sous le logiciel R, et plus précisément du package TraMineR, développé par Gabadinho et al., 2009)(*).

Les Méthodes d'Appariement Optimal permettent de comparer le degré de similarité de séquences. Elles bâtissent alors des typologies de séquences, c'est-à-dire qu'elles rapprochent des suites d'éléments. Le calcul d'une distance entre chaque individu s'opère en fonction du nombre de transformations nécessaires pour passer d'une séquence à une autre. Le résultat se présente sous la forme d'une matrice symétrique de distances.

Une fois que la matrice a été calculée, une méthode de regroupement est appliquée pour agréger les séquences en un nombre réduit de groupes.

(*) : Gabadinho A., Studer M., Müller N., Ritschard G. (2009) : http://mephisto.unige.ch/traminer/

Nous revenons ici plus en détail sur les cinq trajectoires types.

\section{-Trajectoire 1 : formation générale puis études supérieures longues (50\%)}

Cette trajectoire est assez linéaire : les individus sont au collège pendant quatre ans puis obtiennent un baccalauréat général et poursuivent ensuite des études longues (type universitaire ou grandes Ecoles). La plupart d'entre eux sont donc encore en études en 2007 (68\%).

Au sein de cette trajectoire, les pères des individus possèdent les plus hauts niveaux d'éducation (29 \% possèdent comme diplôme le plus élevé un diplôme universitaire de deuxième ou troisième cycle). Par ailleurs, seulement $10 \%$ des individus ont un père dont le diplôme le plus élevé est un certificat d'études primaires (contre par exemple 28 \% pour les individus de la trajectoire 5$)^{3}$. D'une certaine façon, ce constat semble faire écho à la notion de démocratisation ségrégative de Merle (2002). Autrement dit, l'élargissement de l'accès aux études longues ne modifie pas les parts respectives de chaque groupe social.

3. Nous avons fait le choix de nous intéresser au niveau de diplôme du père plutôt qu’à la CSP (catégorie sociale et professionnelle) car nous travaillons sur les trajectoires scolaires des jeunes. Autrement dit, la CSP ne permet pas, selon nous, d'estimer au mieux l'environnement culturel des jeunes : le père peut avoir connu soit un déclassement sur le marché du travail par rapport à son niveau d'études, soit au contraire une "promotion ». De ce fait, la CSP ne permet pas d'appréhender systématiquement les ressources culturelles du père qui peuvent s'avérer déterminantes dans une trajectoire (aide aux devoirs...). 


\section{-Trajectoire 2 : formation technologique, études supérieures courtes puis entrée sur le marché du travail (21\%)}

Dans cette trajectoire, les individus optent, après le collège, pour une formation technique. Après l'obtention de leur baccalauréat technologique, ils choisissent des études supérieures courtes de type brevet de technicien supérieur (BTS), voire diplôme universitaire de technologie (DUT). Les pères possèdent des diplômes moins élevés que précédemment puisque seulement $6 \%$ des individus ont un père dont le diplôme le plus élevé est un diplôme universitaire de deuxième ou troisième cycle. Ainsi l'environnement culturel continue de peser sur le choix des filières d'études. Si les jeunes issus des milieux moins favorisés culturellement accèdent à l'enseignement supérieur, ils s'orientent cependant dans certaines filières moins prisées par les catégories plus favorisées (Bourdieu et Passeron, 1964).

\section{-Trajectoire 3 : formation professionnelle, études supérieures courtes puis entrée sur le marché du travail (9\%)}

Cette trajectoire est un peu moins linéaire que précédemment. En premier lieu, il faut noter que le collège dure ici cinq ans (la plupart des individus redoublent une année avant d'entrer dans le secondaire supérieur). Ensuite, la majorité des élèves optent pour une formation de type CAP-BEP puis entrent en baccalauréat professionnel. Ils obtiennent ainsi le " bac » à environ 20 ans (au lieu de 18 ans dans la première trajectoire). Après l'obtention de ce dernier, la majorité d'entre eux tente des études supérieures courtes (professionnelles, type BTS). Soulignons que pour cette trajectoire, très rares sont ceux qui s'insèrent directement sur le marché du travail après le baccalauréat. "Alors même que le baccalauréat professionnel a pour vocation première de préparer à l'entrée immédiate sur le marché du travail, une proportion non négligeable de détenteurs de ce diplôme choisissent d'entrer dans l'enseignement supérieur, essentiellement dans des fliières professionnelles " (Moullet, 2005). Cette trajectoire est également celle où les pères ont les diplômes les moins élevés (seulement $3 \%$ d'entre eux détiennent un diplôme universitaire de deuxième ou troisième cycle et $5 \%$ ont un diplôme du supérieur de type premier cycle ou de type BTS ou DUT).

\section{-Trajectoire 4 : formation générale, études supérieures courtes puis entrée sur le marché du travail (16\%)}

Cette trajectoire est assez linéaire. Les élèves suivent des études au collège pendant quatre ans puis entrent au lycée pour obtenir un baccalauréat général. Ils optent ensuite pour une formation supérieure courte (avec des études de type BTS ou IUT, voire licence). Parmi les trajectoires à études courtes, il s’agit du parcours où les pères détiennent les diplômes les plus élevés ( $12 \%$ d'entre eux ont un diplôme universitaire de deuxième ou troisième cycle et $14 \%$ ont un diplôme du supérieur de type premier cycle ou de type BTS ou DUT). 


\section{-Trajectoire 5 : formation professionnelle et technologique, études supérieures courtes puis entrée sur le marché du travail (4\%)}

Cette trajectoire est assez proche de la trajectoire 3, à la différence près qu'après une formation professionnelle type CAP-BEP, les individus intègrent une formation secondaire plus technique, type baccalauréat technologique ou brevet technique. Le collège dure également cinq ans. Les pères possèdent des diplômes assez faibles (seulement $3 \%$ d'entre eux détiennent un diplôme universitaire de deuxième ou troisième cycle et $9 \%$ sont titulaires d'un diplôme du supérieur de type premier cycle ou de type BTS ou DUT).

\section{Quel espace des possibles pour quelle trajectoire?}

Les variables classiques telles que le sexe, l'origine sociale des parents, pour n'en citer que deux, sont certes des facteurs permettant d'éclairer les trajectoires de formation et d'emploi ; cependant, il convient d'aller au-delà de ces facteurs en cherchant à contextualiser au mieux les univers dans lesquels les acteurs sociaux évoluent. La variable « niveau d'études du père » joue certes un rôle sur la probabilité d'appartenance à une trajectoire-type ; pour autant, le niveau d'études du père se combine à d'autres variables, produisant ainsi des contextes plus ou moins favorables à tel ou tel type de trajectoire scolaire. L'analyse de ces contextes permet de mieux comprendre les trajectoires différenciées (Lahire, 1995). Ainsi certains facteurs, tels que le niveau d'étude du père, le fait d'avoir redoublé au primaire ou encore l'aide aux devoirs de la part des parents, se cumulent et ouvrent ou ferment l'espace des possibles pour les acteurs sociaux.

\subsection{Des chances inégales d'appartenance à une trajectoire-type}

Dans cette partie, nous tentons de déterminer les chances d'appartenance à telle trajectoire type plutôt qu'à telle autre à l'aide d'un modèle logistique. L'objectif est alors d'apprécier les caractéristiques des personnes appartenant aux différentes trajectoires et d'évaluer leur marge de manœuvre par rapport à leur environnement socio-culturel. Nous considérons alors plusieurs dimensions de l'environnement familial afin de spécifier l'environnement socio-économique et culturel des individus. En premier lieu, d'une manière assez classique, nous prenons en compte le niveau d'études du père ${ }^{4}$ et son pays de naissance. Pour évaluer le soutien de la famille, nous considérons également si l'individu a reçu ou non de l'aide pour le travail scolaire lors du secondaire inférieur et si la famille juge les ressources suffisantes pour une poursuite d'études. Nous prenons aussi en compte le fait d'être ou non inscrit en début de parcours dans une bibliothèque. Cette variable peut être consi-

4. Pour des raisons de lisibilité de l'article, nous avons retenu le niveau d'études d'un seul parent, en l'occurrence celui du père, qui est dans notre étude davantage significatif que celui de la mère. 
dérée comme un indicateur approché (proxy) d'un environnement culturel soit plutôt favorable soit plutôt défavorable à l'éducation.

Afin de caractériser l'impact de la situation éducative en début de parcours de l'individu, nous considérons son niveau en mathématiques ${ }^{5}$ en début de secondaire inférieur. Cela nous permet d'apprécier la marge de manœuvre de l'individu entre ses résultats au début du collège et sa trajectoire de formation, d'insertion et d'entrée dans la vie adulte et donc le degré de réversibilité des trajectoires. Pour définir la situation en début de trajectoire, nous considérons également le fait que l'individu a déjà redoublé ou pas.

Nous contrôlons le sexe et le fait que l'individu a fait ou non partie d'une Zone d'Education Prioritaire (ZEP) en début de trajectoire.

Dans le modèle, la variable explicative est le fait d'appartenir à une trajectoire x par rapport au fait d'appartenir à la trajectoire 1 (formation générale puis études supérieures longues), qui constitue la trajectoire rassemblant le plus grand nombre.

\section{Encadré 3}

\section{Le modèle multinomial}

L'objet est d'identifier, parmi les caractéristiques individuelles, en particulier celles relatives au niveau de formation du père, à l'environnement culturel de la famille de l'individu et aux performances scolaires en mathématiques, les déterminants de l'appartenance à l'une ou l'autre des cinq trajectoires mises en évidence. On s'intéresse donc aux déterminants de l'appartenance aux trajectoires-types précédemment établies. Dans cette perspective, nous estimons un modèle logit multinomial (Gouriéroux, 1984) dans lequel la typologie (cinq modalités) est la variable à expliquer et les caractéristiques individuelles constituent les facteurs explicatifs. II s'agit d'un modèle logit polytomiques non ordonnés. Nous raisonnons donc sous hypothèse d'indépendance des choix offerts (IIA : Independance from Irrelevant Alternatives) : à savoir que le modèle logit ne prend pas en compte la proximité de nature qui peut exister entre plusieurs choix offerts à l'individu. Il est structuré de manière telle que l'individu arbitre entre deux choix $a$ et $b$, indépendamment des autres choix qui lui sont offerts.

5. Il s'agit d'une note qui doit apprécier le niveau de l'élève en mathématiques à son entrée au collège par rapport à ce que l'on attend d'un élève parvenu en Ge. 
Tableau 1

Résultats du modèle multinomial en pourcentages

\begin{tabular}{|c|c|c|c|c|}
\hline & \multicolumn{4}{|c|}{ Rapports de risques relatifs } \\
\hline Trajectoires & $\begin{array}{l}\text { Formation techno- } \\
\text { logique, études sup } \\
\text { courtes }\end{array}$ & $\begin{array}{l}\text { Formation profes- } \\
\text { sionnelle, études } \\
\text { sup courtes } \\
\text { sus Formation gér }\end{array}$ & $\begin{array}{l}\text { Formation générale, } \\
\text { études sup courtes }\end{array}$ & $\begin{array}{l}\text { Formation profes- } \\
\text { sionnelle et techno- } \\
\text { logique, études sup } \\
\text { courtes } \\
\text { ues }\end{array}$ \\
\hline $\begin{array}{l}\text { Variables: } \\
\text { Sexe : hommes }\end{array}$ & 117 & 273,1 & ns & 270,4 \\
\hline Diplôme le plus élevé du père : (ref: CAP-BEP) & & & & \\
\hline Aucun diplôme/ Certificat d'études primaires & ns & ns & ns & 90,4 \\
\hline Brevet élémentaire, brevet des collèges & ns & ns & ns & ns \\
\hline Baccalauréat général & $-61,4$ & ns & $-37,5$ & $-79,2$ \\
\hline $\begin{array}{l}\text { Baccalauréat technologique ou professionnel, } \\
\text { brevet professionnel }\end{array}$ & $-53,6$ & $-49,7$ & $-39,7$ & ns \\
\hline Diplôme universitaire de 1er cycle, BTS, DUT & $-67,7$ & $-79,1$ & $-41,1$ & $-59,9$ \\
\hline Diplôme universitaire de Zème ou 3ème cycle & $-87,4$ & $-94,7$ & $-72,8$ & $-91,3$ \\
\hline Pays de naissance du père : (ref :France) & & & & \\
\hline DOM-TOM & ns & ns & ns & ns \\
\hline Etranger & ns & ns & ns & ns \\
\hline $\begin{array}{l}\text { Adéquation entre les ressources de la famille } \\
\text { projets d'études: (ref: juste suffisant) } \\
\text { très insuffisant }\end{array}$ & ns & ns & ns & ns \\
\hline un peu insuffisant & ns & ns & ns & ns \\
\hline tout à fait suffisant & ns & ns & $-28,4$ & $-51,3$ \\
\hline Aide à la maison pour le travail scolaire: non & $-31,3$ & $-56,7$ & $-28,7$ & $-79,2$ \\
\hline ZEP en 1995 & 55 & ns & ns & ns \\
\hline Redoublement au primaire & 551,8 & 7,5 & ns & 2822,2 \\
\hline Fréquentation d'une bibliothèque: non & 31,2 & 72,4 & 49,4 & ns \\
\hline Résultats en mathématiques & $-39,8$ & -66 & $-20,6$ & $-62,2$ \\
\hline
\end{tabular}

Source : panel DEPP-EVA.

Les résultats présents dans le tableau sont tous significatifs. Les chiffres en gras indiquent des résultats significatifs au seuil de 1\% et 0,1\%. Les chiffres en italique indiquent des résultats significatifs au seuil de $5 \%$.

Note de lecture : les Rapports de Risques Relatifs (Relative Risk Ratios) représentent le rapport entre la probabilité d'appartenance à la trajectoire jet la probabilité d'appartenance à la trajectoire 1 ; ils permettent de faciliter l'interprétation. Ainsi un individu ayant un père possédant comme diplôme le plus élevé un baccalauréat général a $61 \%$ de risque en moins d'être dans la trajectoire 2 par rapport à la trajectoire 1.

Sigles : CAP-BEP : certificat d'aptitude professionnelle et brevet d'études professionnelles ; BTS et DUT : brevet de technicien supérieur et diplôme universitaire de technologie. ZEP : zone d'éducation prioritaire. DOM-TOM : départements et territoires d'Outre-mer. 
Nous remarquons qu'il n'existe pas de différence entre trajectoires au regard de l'adéquation entre les ressources de la famille et les projets d'études. Cette variable est en effet peu significative. Nous pouvons en outre faire l'hypothèse que les familles ont déjà en quelque sorte " rationnaliser " les ambitions scolaires qu'elles peuvent avoir pour leurs enfants en fonction de leurs ressources. Il pourrait donc s'agir de ce que Sen appelle une situation de "préférences adaptatives ", c'est-à-dire une situation où les individus ajustent leurs préférences en fonction de ce qui leur parait " réaliste ".

Par ailleurs, il est surprenant de constater que, toutes choses égales par ailleurs, le rôle du pays de naissance du père n'a aucun impact sur l'appartenance aux trajectoires. Il semblerait alors que le niveau d'éducation du père fasse davantage la différence.

Concernant les différences entre la trajectoire 2 (études technologiques puis études courtes) et la trajectoire 1 (trajectoire académique linéaire), nous observons de grandes disparités relatives au diplôme du père : un individu dont le père possède comme diplôme le plus élevé un baccalauréat général a $61 \%$ de risque en moins d'être dans cette trajectoire et un individu dont le père détient, comme diplôme le plus élevé, un diplôme universitaire de deuxième ou troisième cycle a $87 \%$ de risque en moins de se retrouver dans cette trajectoire. L'absence d'aide aux devoirs de la part des parents au collège diminue les risques d'être dans cette trajectoire ; les jeunes ayant connu ce parcours rencontreraient des difficultés scolaires et seraient donc davantage aidés par leurs parents (les individus sans aide au collège ayant ainsi plus de chance de se retrouver dans le parcours d'études supérieures longues). La situation scolaire en début de secondaire joue un rôle très significatif sur la trajectoire. En effet, un individu ayant redoublé une classe au primaire a $552 \%$ de risque en plus d'être dans cette trajectoire et le fait d'avoir une meilleure note en mathématiques diminue de $40 \%$ le risque de se retrouver dans celle-ci.

Quant aux risques d'appartenir à la troisième trajectoire (trajectoire professionnelle), les garçons ont $273 \%$ de risque d'être ici plutôt que dans la trajectoire académique (trajectoire de référence). Avoir un père avec un haut niveau de diplôme joue encore un rôle considérable. Ainsi un individu dont le père possède comme niveau de diplôme le plus élevé un diplôme universitaire de deuxième ou troisième cycle a $95 \%$ de risque en moins de se retrouver dans cette trajectoire professionnelle. Comme précédemment, le fait que les parents n'aident pas l'enfant au collège diminue les risques d'être dans cette trajectoire.

En revanche, le fait de ne pas fréquenter une bibliothèque augmente significativement les risques d'être dans cette trajectoire. Enfin, la situation scolaire en début de secondaire joue ici aussi un rôle très significatif sur la trajectoire : un individu ayant redoublé une classe au primaire a presque 11 fois plus de risque de se retrouver dans cette trajectoire ; en revanche, avoir une meilleure note en mathématiques diminue de $66 \%$ le risque de s'y trouver. 
S'agissant de la différence entre la trajectoire 4 (secondaire général puis études supérieures courtes) et la trajectoire de référence (formation générales puis études supérieures longues), le genre semble n'avoir aucun rôle. En revanche, les diplômes des pères continuent d'avoir ici un impact significatif sur la trajectoire. Ainsi de manière très significative, un individu dont le père possède comme diplôme le plus élevé un diplôme universitaire de deuxième ou troisième cycle a $73 \%$ de risque en moins de se retrouver dans cette trajectoire. Par ailleurs, il semble qu'il y ait une certaine différence entre cette trajectoire et la trajectoire d'études longues et académiques (trajectoire de référence) du point de vue de la fréquentation d'une bibliothèque dans le secondaire : en effet, le fait de ne pas être inscrit dans une bibliothèque augmente de $49 \%$ les risques d'être dans cette trajectoire. Ce résultat semble valider en partie notre hypothèse de la fréquentation d'une bibliothèque comme indicateur approché (proxy) d'un certain capital culturel. Enfin, si le fait d'avoir redoublé ne joue aucun rôle ici, la performance scolaire conserve un rôle très significatif: le fait d'avoir de bonnes notes en mathématiques diminue de $21 \%$ les risques d'appartenir à cette trajectoire

Enfin, pour la dernière trajectoire (trajectoire professionnelle et technologique puis entrée sur le marché du travail), les garçons ont également plus de risque d'appartenir à cette trajectoire. Le fait que les individus aient des pères avec des hauts nivaux d'éducation joue toujours un rôle très important. En effet, un individu dont le père possède comme diplôme le plus élevé un diplôme universitaire de deuxième ou troisième cycle a $91 \%$ de risque en moins de se retrouver dans cette trajectoire. Comme dans la première trajectoire professionnelle, l'absence d'aide aux devoirs de la part des parents au collège diminue les risques d'être dans cette trajectoire. La situation scolaire en début de secondaire joue ici aussi un rôle considérable sur la trajectoire : un individu ayant redoublé une classe au primaire a presque 29 fois plus de risque d'être dans cette trajectoire professionnelle ; avoir une meilleure note en mathématiques diminue de $62 \%$ le risque de se retrouver dans celle-ci.

Ainsi l'appartenance à une trajectoire type semble largement influencée par l'environnement socioculturel de l'individu. Un père possédant un haut niveau de diplôme par exemple diminue très significativement les risques d'appartenir aux formations plus professionnelles. De même, le fait de ne pas être inscrit dans une bibliothèque en début de parcours augmente les risques d'être dans un parcours professionnel. Par ailleurs, le début de la scolarité de l'individu semble surdéterminer sa trajectoire ; l'individu ayant commencé sa scolarité avec des difficultés (redoublement au primaire et mauvaise note en mathématiques) n'a quasiment pas de marge de manœuvre. Certaines trajectoires paraissent donc relativement irréversibles. Au-delà du fait que les individus ne sont pas tous égaux face à l'appartenance à une trajectoire type, il faut remarquer que cette appartenance peut également venir influencer l'espace des possibles des individus. Nous nous focaliserons ainsi sur l'espace des possibles et les contraintes associées à chaque trajectoire. 


\subsection{Les trajectoires de formation et d'entrée dans la vie adulte déterminent les opportunités}

L'appartenance à une trajectoire type est d'autant plus importante que les différentes trajectoires ne sont pas nécessairement associées aux mêmes contraintes et aux mêmes opportunités. Deux dimensions sont alors abordées ici, les contraintes et les opportunités à l'intérieur de la trajectoire de formation et celles, dans les dernières années, sur le marché du travail et plus largement lors de l'entrée dans la vie adulte. Nous étudions d'abord deux périodes charnières des trajectoires de formation des individus : l'orientation et l'arrêt des études. Ensuite, nous revenons sur des réalisations importantes en termes de passage à l'âge adulte (type d'emploi, situation financière, décohabitation). Les résultats qui suivent s'appuient sur l'enquête Jeunes de 2002 pour la première partie et sur l'enquête EVA (2004, 2005, 2006 et 2007) pour la seconde partie. Les résultats suivants ont été obtenus par le croisement entre les différentes trajectoires et les situations aux années données précédemment.

Nous nous concentrons alors sur les trajectoires de formation et sur l'entrée dans la vie adulte, chacune étant appréhendée à partir de différents indicateurs permettant de renseigner sur l'espace des possibles des individus.

\subsubsection{Des trajectoires de formation plus ou moins contraintes}

La notion de choix réel est fondamentale dans une analyse par les " capabilités ». En effet, une trajectoire réellement " capabilisante " serait une trajectoire non seulement nondéterminée et réversible à tout moment mais aussi et surtout véritablement choisie par l'individu. Parler de choix en éducation, notamment les choix éducatifs opérés dans le secondaire, est un peu délicat, notamment au niveau de la responsabilité individuelle, puisqu'il s'agit de choix faits, en grande partie, par des mineurs (Saito, 2003). La première variable prise en compte ici est en fait le vœu d'orientation formulé par la famille de l'individu, ce vœu reflétant peu ou prou la marge de manœuvre de l'individu. Existe-t-il des trajectoires de formation plus contraintes que d'autres? Il s'agit ici d'analyser l'aspect choix de ces trajectoires ; à cet effet, nous avons sélectionné deux autres variables, le refus d'un vœu d'orientation et le motif d'arrêt des études.

Lorsque nous nous intéressons au premier vœu d'orientation formulé par la famille en fin de $3^{\text {ème }}$, nous constatons que $55 \%$ des jeunes de la trajectoire 5 (professionnelle et technologique puis entrée sur le marché du travail) et $50 \%$ de ceux de la trajectoire 3 (professionnelle puis études courtes et entrée sur le marché du travail) n’ont pas été orientés selon leur premier vœu, alors que l'orientation des jeunes de la trajectoire 1 (formation générale puis études supérieures longues) correspond à leur premier vœu d'orientation (99\%).

Ces données sont corroborées par la variable rétrospective de l'enquête Jeunes 2002 «refus d'un vœu d'orientation lors de la scolarité ", puisque seuls $66 \%$ des jeunes appartenant à la trajectoire 3 (professionnelle) et $55 \%$ des jeunes appartenant à la trajectoire 5 
(professionnelle puis technologique) considèrent avoir toujours été orientés selon leurs vœux lors de leur scolarité, contre $95 \%$ des jeunes de la trajectoire 1 (études supérieures longues) et $80 \%$ de ceux de la trajectoire 4 (études supérieures courtes). Ainsi pour les jeunes de la trajectoire professionnelle et technologique, $45 \%$ des orientations sont subies et ne résultent donc pas d'un véritable choix (contre $5 \%$ de choix subis dans les parcours d'études longues). Nous retrouvons l'idée que ces filières d'études, en France, sont considérées comme des filières par défaut, au sein desquelles l'orientation peut s’opérer de manière véritablement contrainte. Les trajectoires 3 (professionnelle) et 5 (professionnelle et technologique) semblent donc, de ce point de vue, moins «capabilisantes » que la trajectoire 1 (formation générale et études supérieures longues), dans le sens où elles résultent moins souvent d'un véritable choix ${ }^{6}$.

La contrainte en termes de choix est renforcée par l'analyse de la variable " arrêt des études ». En effet, parmi les jeunes qui ne poursuivent plus d'études au $1^{\text {er }}$ mars 2007, $17 \%$ de ceux qui appartiennent à la trajectoire 5 (professionnelle et technologique) ont arrêté leurs études pour des raisons financières, contre $9 \%$ de ceux de la trajectoire 4 (universitaire court) et $0 \%$ de la trajectoire 1 (général et études supérieures longues). En outre, $17 \%$ des jeunes de la trajectoire 5 (professionnelle et technologique) ont arrêté leurs études en raison d'un refus de leur dossier, contre $0 \%$ des jeunes de la trajectoire 1.

La contrainte qui pèse sur les choix de formation semble induire un pessimisme plus fort vis-à-vis de l'avenir professionnel puisque sept ans après l'entrée en sixième, $27 \%$ des jeunes de la trajectoire 5 (professionnelle et technologique) sont plutôt inquiets par rapport à leur avenir professionnel contre $18 \%$ des jeunes de la trajectoire 1 (général et études supérieures longues). Ce phénomène corrobore l'étude menée par De Besses (2007) montrant qu'une orientation subie affecte la confiance que l'individu a en luimême et en l'avenir.

Ainsi certains parcours semblent cumuler irréversibilité et non-choix. Nous allons revenir sur leur situation professionnelle cinq ans après. En effet, quelles sont réellement leurs situations en 2007 sur le marché du travail, et plus largement en termes d'entrée dans la vie adulte?

\subsubsection{Le passage à la vie adulte : quels espaces des possibles ?}

Dans cette partie, nous nous focalisons sur l'espace des possibles des personnes lors du passage à la vie adulte pour chaque trajectoire.

Lorsque nous observons la situation vis-à-vis de l'emploi des jeunes des différentes trajectoires, parmi les quatre trajectoires où les individus sont entrés sur le marché du travail, on n'observe pas de différences significatives en matière de contrat de travail. En effet,

6. Voir le numéro 109 de Formation Emploi « L'orientation scolaire et professionnelle dans un monde incertain », janvier-mars 2010. 
$20 \%$ des jeunes de la trajectoire 2 (technologique et études supérieures courtes) sont en contrat à durée déterminée (CDD) et $26 \%$ des jeunes de la trajectoire 5 (professionnelle et technologique) sont dans ce cas. Toutefois, ceux des trajectoires 3 (professionnel) et 4 (supérieur court) sont seulement $19 \%$ à être en CDD.

En ce qui concerne la situation face à l'emploi et plus précisément le fait d'être sans emploi et à la recherche d'un emploi, quelques différences importantes apparaissent : alors qu'ils sont $4 \%$ de la trajectoire 4 (supérieur court), ils sont $9 \%$ de la trajectoire 3 (formation professionnelle). Il convient de préciser, même si le panel DEPP ne permet pas de le mesurer, que les opportunités d'emploi sont variables selon les régions géographiques, et selon la taille des villes. Les enfants de cadres supérieurs vivant davantage dans les grandes villes que les enfants d'ouvriers, leurs opportunités en termes d'emploi sont de ce fait plus grandes.

Par ailleurs, selon leur trajectoire d'appartenance, les jeunes sont plus ou moins nombreux à rencontrer des difficultés financières. En 2007, 28 \% des jeunes de la trajectoire 1 (formation générale et supérieur long) et $29 \%$ des jeunes de la trajectoire 4 (supérieur court) ont rencontré des difficultés financières, contre $40 \%$ de ceux de la trajectoire 5 (formation professionnelle et technologique) et $38 \%$ de ceux de la trajectoire 3 (formation professionnelle). Ainsi l'appartenance à une trajectoire type peut induire des contraintes dans la vie quotidienne, en l'occurrence ici le fait d'être confronté à des difficultés financières.

Les jeunes ne sont pas égaux non plus en matière d'accès à l'enseignement supérieur. Ainsi la répartition des établissements d'enseignement supérieur est inégale sur le territoire. Certains étudiants doivent soit décohabiter pour suivre les études qu'ils souhaitent, soit choisir de manière contrainte une filière à proximité du domicile parental. De cette manière, la formation suivie peut alors résulter davantage d'un choix contraint que d'un véritable choix. 
En 2004, les jeunes de la trajectoire 1 (cursus universitaire long et linéaire) sont pour $64 \%$ d'entre eux décohabitants, alors qu'ils sont seulement $35 \%$ de la trajectoire 3 (formation professionnelle) et $37 \%$ de la trajectoire 5 (formation professionnelle et technologique) ${ }^{7}$. Si les jeunes des formations techniques et professionnelles décohabitent moins, cela peut s'expliquer par le fait que ces formations supérieures de type professionnel sont davantage présentes sur le territoire français que des classes préparatoires ou même un premier cycle universitaire. Ces différences persistent malgré la stabilisation en emploi. En 2007, alors même que les jeunes de la trajectoire 5 (formation professionnelle et technologique) et ceux de la trajectoire 3 (formation professionnelle) sont plus nombreux à être titulaires d'un contrat à durée indéterminée (CDI) que ceux de la trajectoire 18 (formation générale et supérieur long) (respectivement $60 \%$ et $69 \%$ vs $45 \%$ ), ils demeurent plus souvent chez leurs parents (respectivement $43 \%$ et $49 \%$ vs $26 \%$ ). Ainsi paradoxalement, l'emploi, notamment l'emploi stable, n'apparaît donc pas comme une condition d'accès à l'autonomie résidentielle. Certains parcours de formation sont donc plus restrictifs sur le champ des possibles du point de vue de l'indépendance résidentielle (Farvaque, Oliveau, 2004).

\section{Conclusion}

L'objectif de ce travail était d'analyser les parcours de formation des individus à l'aune de la théorie des "capabilités " de Sen, afin de rendre compte de l'espace des possibles des individus. Un modèle multinomial nous a conduits à déterminer les caractéristiques des personnes appartenant à telle ou telle trajectoire. Il nous a permis de montrer que des éléments tels que le niveau de diplôme du père et l'inscription ou non dans une bibliothèque jouaient, au départ, un rôle significatif sur l'affectation à une trajectoire. En outre, nous avons observé que les résultats à l'entrée dans l'enseignement secondaire et un redoublement au primaire jouaient également un rôle déterminant. Ces éléments nous révèlent que certains individus disposent d'une marge de manœuvre assez limitée dans leur trajectoire, et ce dès le début.

Dans un deuxième temps, nous nous sommes efforcées de mettre en exergue l'influence de l'appartenance à une trajectoire type sur l'espace des possibles des individus. Nous avons focalisé notre analyse sur les décisions de formation aux périodes charnières : l'orientation et l'arrêt des études. Nous avons ensuite croisé les différentes trajectoires avec deux réalisations cruciales pour le passage à la vie adulte et l'accès à l'autonomie : le logement et l'emploi.

7. Il est possible que certains jeunes, après une première décohabitation parentale, cohabitent à nouveau avec leurs parents ; toutefois, ici nous cherchons à comparer la situation des jeunes des différentes trajectoires en matière de logement à un temps t, quelle que soit leur situation résidentielle antérieure.

8. Un certain nombre des jeunes de la trajectoire 1 sont encore en études ou effectuent des stages. 
Ce travail montre ainsi que des difficultés scolaires et un environnement socioculturel défavorisé peuvent sanctionner l'ensemble d'une trajectoire ; la conjugaison de ces éléments pouvant conduire à des formations généralement davantage subies que choisies, et plus tard à des difficultés accrues lors des réalisations importantes du passage à la vie adulte (difficultés financières, reprise de la cohabitation avec les parents malgré la stabilité dans l'emploi). Pour certaines personnes, les contraintes semblent donc se cumuler dans le temps.

La liberté de choix dans le système éducatif doit ainsi être appréhendée d'une façon large car le degré de liberté dans l'éducation conditionne les opportunités futures de l'individu dans sa vie d'adulte. Saito (2003) rappelle que le rôle de l'éducation est primordial en matière de "capabilités "; elle renforce en effet l'autonomie de l'individu et donc d'une certaine manière libère ses choix par rapport à son environnement (particulièrement ici l'environnement familial). Dès lors, l'institution scolaire apparait comme la clef de voûte conditionnant l'étendue des libertés des personnes.

Un système scolaire pleinement « capabilisant » devrait alors libérer les individus des déterminismes socio-économiques. Il doit s'assurer qu'une multitude de choix est possible pour l'ensemble des personnes et favoriser la réversibilité de ces choix. Certains dispositifs scolaires peuvent alors renforcer ces possibilités, comme un véritable tronc commun lors du collège (laissant plus de temps aux individus pour se révéler) ou le renforcement du rôle des professionnels de la régulation intermédiaire (rôle des conseillers d'orientation dans le soutien à la formulation d'un vœu, rôle des accompagnateurs pédagogiques, etc.), lors de la première orientation mais aussi tout au long du parcours de formation.

Nos résultats montrent en outre que l'irréversibilité des parcours de formation débute bien avant la première orientation, la situation scolaire en Gème étant déterminante pour le parcours de l'individu. Ce constat pose la question du rôle du collège unique en France. Il semblerait ici que les possibilités que ce dispositif est censé ouvrir soient plus formelles que réelles.

\section{Bibliographie}

Ammermüller A. (2005), "Educationnal opportunities and the role of the institutions: an international comparison”, ESPE annual Conference, Mannheim.

Bourdieu P., Passeron J.-C. (1964), Les Héritiers, les étudiants et la culture, Paris, Les Editions de Minuit.

Bourdieu P., Passeron J.-C. (1970), La reproduction. Eléments pour une théorie du système d'enseignement, Paris, Minuit.

Besses (DE) M. (2007), "L'estime de soi, enjeu éducatif pour préparer à la vie professionnelle ", in Formation Emploi, n ${ }^{\circ} 98$. 
Duru-Bellat M. (2002), Les inégalités sociales à l'école : genèse et mythes, Paris, PUF.

Farvaque N., Oliveau J.-B. (2004), L'insertion des jeunes peu diplômés dans l'emploi : opportunités de choix et contraintes, Documents de travail Institutions et Dynamiques Historiques de l'Economie.

Formation Emploi (2010), Dossier "L'orientation scolaire et professionnelle dans un monde incertain $" \mathrm{n}^{\circ \circ} 109$.

Gabadinho A., Ritschard G., Studer M. et Müller N.S. (2009), Mining Sequence Data in R with the TraMineR package: A User's Guide, Department of Econometrics and Laboratory of Demography, University of Geneva, Geneva.

Gouriéroux C. (1984), Econométrie des variables qualitatives, Coll. " Economie et statistiques avancés ", Economica.

Lahire B. (1995), Tableaux de famille. Heurs et malheurs scolaires en milieux populaires, Paris, Gallimard/Seuil, coll. "Hautes Etudes".

Maurice M., Sellier F. et J.-J. Silvestre, (1982), Politique d'éducation et organisation industrielle en France et en Allemagne : essai d'analyse sociétale, Paris, PUF.

Merle P. (2002), La démocratisation de l'enseignement, Paris, La Découverte.

Mons N. (2004), De l'école unifiée aux écoles plurielles : évaluation internationale des politiques de différenciation et de diversification de l'offre éducative, thèse de doctorat inédite en sciences de l'éducation, université de Bourgogne.

Moullet S. (2005), «Après le bac professionnel ou technologique : la poursuite d'études jusqu'à bac +2 et sa rentabilité salariale en début de vie active ", Economie et Statistique, $n^{\circ} 388-389$.

Otтo H.U. and Ziegler H. (2006), "Capabilities and Education”, in Social Work and Society, Volume 4, n 2.

Robeyns I. (2000), "An unworable idea or a promising alterbative? Sen's capbility approach re-examined", mimeo, Wolfson College, université de Cambridge.

Saito M. (2003) “Amartya Sen’s Capability Approach to Education: A Critical Exploration", in Journal of Philosophy of Education, Volume 37, $\mathrm{n}^{\circ} 1$

Sen A. (1985), Commodities and Capabilities, Londres, Elsevier Science Publishing Company.

SEn A. (1992), Inequality Re-examined, Harvard, Harvard University Press.

SEN A. (1993), Ethique et économie, Paris, PUF.

Verdier E. (2008), «L'éducation et la formation tout au long de la vie : une orientation européenne, des régimes d'action publique et des modèles nationaux en évolution ", Sociologie et Sociétés, Vol. XL.1. 
Verhoeven M., Orianne J.-F. et Dupriez V.(2007), « Vers des politiques d'éducation "capacitantes "? ", in Formation Emploi, no 98.

Woesmann L. (2004), "How Equal are Educational Opportunities? Family Background and Student Achievement in Europe and the United States", CESifo Working Paper, 1162. 